\title{
Sociedad, Universidad, Empresa y Talento en momentos de cambio. Aprender y desaprender para volver a emprender.
}

\author{
Vanessa Argüelles Rodríguez \\ vanessaarguelleslapfsgrupo.com \\ Universidad de Oviedo - PFSGrupo (España) \\ ORCID: https://orcid.org/0000-0003-3761-5386
}

\section{RESUMEN}

Universidad, Empresa y Sociedad vienen guardando una estrecha relación condicionada por los elementos económicos, sociales, políticos y funcionales que operan en cada momento histórico; hoy, la cultura academicista y una visión funcionalista de la adquisición de competencias y habilidades de liderazgo tratan de convivir y engranarse en los entornos universitarios. Este artículo, elaborado en base a una revisión documental, analiza el concepto de talento desde el foco de las empresas y estudia la permuta hacia modelos de estudiantes favorecedores de empleados más preparados para trabajar, con aspiraciones y protagonistas de su propio aprendizaje para disponer de más oportunidades que mejoren su empleabilidad y, por ende, su calidad de vida. Esta investigación invita a la reflexión de si las universidades deben asumir un nuevo enfoque para adaptarse a un contexto emergente y prever situaciones futuras que las mantengan como agentes y referentes educativos.

Palabras Clave: aprendizaje, enseñanza, formación, talento, competencia y liderazgo. 


\section{Introducción}

En la actual sociedad capitalista, los índices de empleabilidad de nuestro país afectan de continuo a todas las áreas de nuestras vidas creciendo paulatinamente, según el Centro de Investigación Sociológicas (CIS, 2020), la preocupación de los ciudadanos por el ámbito económico, por las cifras del paro y por la precariedad de los puestos de trabajo. El paro sigue siendo el principal problema para cada vez más ciudadanos, incremento que coincide con la publicación a principios de febrero de los datos que señalan que el mes de enero 2020 ha cerrado con más de 90.000 nuevos inscritos en las oficinas públicas de empleo, el peor arranque de año desde el 2013, en España. Todo esto suscita un interrogante constante en la sociedad y en las instituciones que guía, en parte, este estudio sobre las nuevas competencias necesarias para satisfacer la actual demanda laboral.

Se conciben sociedad, empleo y educación como acepciones interconectadas a lo largo de la historia pero que se encuentran en un escenario cambiante, interrelacionado, tecnológico y global al que deben adaptarse para sostenerse de manera favorable. Se alude constantemente a la necesidad de contar con personas talentosas, a la importancia del engranaje entre el sistema educativo y el sistema productivo, al rol de la Universidad como aval de ese talento (Álvarez-Arregui y Arreguit, 2019). Así surgen las primeras evidencias que responden a los objetivos de esta investigación: saber qué entienden por talento las organizaciones laborales, catalogar las competencias que demanda actualmente el mercado laboral, conocer la importancia de la estabilidad laboral para los sistemas y, sobre todo, para las personas y, por último, proponer un modelo universitario acorde al nuevo escenario.

\section{Aproximación conceptual al talento}

Para llegar a conclusiones objetivables y válidas, el primer paso es definir qué se entiende por Talento desde la perspectiva o enfoque que se considera en esta investigación documental, el espacio laboral, a fin de materializarlo en aquellas competencias y habilidades claves para su desarrollo e identificadas y relacionadas con el saber, el saber hacer, el querer hacer y el ser. Dado que en la literatura académica no existe un consenso en lo que respecta al término, cada organización delimita lo que considera un perfil talentoso de acuerdo a sus necesidades actuales o futuras (Tansley, 2011). De acuerdo con la definición que Jericó, Botín y Medina (2008) han dado al concepto de talento, entendemos éste como el mayor patrimonio de las organizaciones y por tanto como el factor que va a suponer la diferencia estratégica entre la excelencia de las empresas. Aprovecha también para señalar que siendo uno de los recursos más valiosos es a menudo el peor gestionado en los entornos laborales, pero esto sería fruto de una investigación complementaria.

Han sido numerosos estudios los que se han servido del término talento entendiéndolo como ciertas habilidades destacadas en multitud de ciencias como la biología, la psicología, la pedagogía, la sociología, la filosofía y la empresa. Lo interesante para este estudio es abordarlo desde la demanda que están haciendo las organizaciones (Lorenzo, 2006). Asimismo, al talento se le ha relacionado en numerosas ocasiones y de manera errónea o confusa con conceptos tales como la genialidad, la superdotación o incluso el logro excelente atribuyéndolo a sujetos que han destacado en alguna materia o ciencia. Así, Berguer (1997) denominó de esta manera a los sujetos que obtenían un alto nivel de inteligencia fruto de un test de sujetos con talento general; Sisk (1993) había aludido a un talento más determinado que tenía que ver con sujetos que destacaban en el desempeño en test de aptitudes más específicas como la capacidad o habilidad para el liderazgo, entendidos los líderes como personas que utilizan determinadas destrezas, que poseen altos niveles de negociación, disponen y demuestran confianza en sí mismos, responsabilidad, tendencia dominante y susceptibilidad para adaptarse a situaciones nuevas.

En el ámbito laboral, según \ericó (2008) el concepto de talento se centra en el conjunto de habilidades relacionadas con cuatro acepciones, tres de ellas correspondidas con el trabajo, definiendo el talento como la capacidad para obtener resultados notables en el ejercicio de la inteligencia, las aptitudes y las habilidades. Talento por tanto es sinónimo de aptitud, capacidad, habilidades e idoneidad. Poseer talento visto desde este prisma sería estar bien dotado para, ser capaz de, tener dotes brillantes para, ser apto para, ser útil para. Y aquí entran en juego las competencias, pues entendemos que son las que sirven de indicadores para desgranar en acciones lo que se entiende como talento.

Baigorri (2018) afirma que el talento reposa en determinadas condiciones personales (parcialmente heredadas) que se proyectarán en diversos campos de nuestra actividad siendo importantísimo comprender que el talento no se desarrolla de forma espontánea ni aleatoria y no siempre en edades tempranas, siendo el resultado de aplicar el esfuerzo personal, la voluntad y el desarrollo de lo que inicialmente no son más que dudosas potencialidades o competencias. El talento se expandirá al máximo cuando se den circunstancias favorables para ello a nivel familiar, personal, educativo, social y profesional. No puede desarrollarse el talento que no se cultiva, y desarrollarlo lo primero es identificarlo (Touron, 2015).

Aunque ni en la práctica empresarial ni en la literatura académica existe consenso a la hora de definir el talento, sí es posible afirmar que, en términos generales, desde ambos ámbitos se alude a un mismo tipo de perfil competencial, independientemente del nombre específico que le confieran (Hernández Lechuga, 2017). El fraccionamiento, por tanto, se hace siempre en base a competencias que son las que generan resultados excelentes. Habría que clarificar el significado del término desde el punto de vista de las organizaciones laborales que es el que se toma para realizar esta investigación.

\section{Competencias y Habilidades}

La introducción del término competencia data de la década de los años 20 en EEUU pero es el auge laboral de los años 60 y 70 donde surge para quedarse de la mano de David Mc Clelland (1973) y se retoma con fuerza en los 90 con las formulaciones de Goleman (1996) acerca de la inteligencia emocional.

Short (1984) apuntaba distintos puntos de vista para definir y comprender las competencias:

- Como conductas, comportamientos o actos que derivan en un desempeño específico.

- Como un conjunto de conocimientos y habilidades concretas que se deben poner en marcha para realizar determinadas acciones.

- Como la capacidad sumada a las habilidades, comportamientos y conocimientos del individuo. Es un tercero quien valora la competencia de una persona, no atiende a estándares definidos sino cambiantes.

- Como una cualidad o estado del ser, algo holístico donde los comportamientos, conocimientos, habilidades e intenciones derivan del estado del ser. 
Para comprender el concepto de competencia encontramos entre los académicos multitud de definiciones que no se prestan a una interpretación práctica necesaria para las empresas (Mertens, 1998), sin embargo redunda el término en las áreas de RRHH lo que afecta al personal empleado y futuro.

Las competencias, básicamente, son a ojos del mercado laboral las respuestas profesionales que un individuo da a los requerimientos de su puesto de trabajo (entendiendo por puesto de trabajo aquel que tiene una ubicación concreta, una actividad específica, un sector, un entorno socio-político y económico, etc.) (Pérez Escoda y Sabariego Puig 2015).

Para ir dando respuesta a todos estos indicadores o requerimientos, las competencias han ido cambiando, evolucionando, sufriendo varias conceptualizaciones; comenzaron siendo capacidades de las personas para desempeñar una determinada actividad o función en un puesto de trabajo (puntos de vista más centrados en la tarea), a continuación pasaron a ser rasgos y características que tenían los profesionales excelentes que daban resultados brillantes en sus respectivas organizaciones (teorías más centradas en el perfil profesional), aquí comenzó a hablarse de competencias clave para llegar a la excelencia. Por último, se ha tendido más hacia modelos que entienden las competencias de forma holística y compleja donde entra en juego la tarea, la excelencia profesional, la capacitación y desarrollo/formación personal y las habilidades y/o actitud para adaptarse al entorno laboral con mercados cambiantes y globalizados.

En enero de 2019, el Instituto de Estudios Superiores de Empresa, IESE, en su informe "El futuro del empleo y las competencias profesionales del futuro: la perspectiva de las empresas", donde la columna vertebral es el gran reto para paliar la tasa de desempleo en España, analiza las competencias requeridas por 53 empresas destacadas del país para cubrir sus necesidades, empresas que en diciembre de 2017 empleaban a casi medio millón de personas en España y a casi tres millones a nivel mundial. El informe concretó que casi tres de cada cuatro empresas señalan dificultades para cubrir los puestos de trabajo ofertados, carencias tanto a nivel de conocimientos en algunas materias como a nivel de capacidades y actitudes profesionales. Concretamente, las empresas entrevistadas muestran una gran preocupación por el cambio de competencias profesionales que la automatización y la revolución digital están generando (un 72\% de estas empresas considera que la revolución digital tiene un impacto alto o muy alto en el cambio de perfiles requeridos y un 56\% de dichas empresas piensa que el impacto de la automatización es alto o muy alto) a la vez que admiten escasez de competencias de liderazgo, habilidades más relacionadas con la actitud, el esfuerzo, el compromiso, la gestión del cambio, las habilidades sociales y de trabajo en equipo, la gestión del estrés, la orientación hacia objetivos, las denominadas softskills en los entornos empresariales.

El informe recoge que la tendencia de las empresas es la evolución hacia estructuras más planas y transversales, con modos de trabajar distintos, que incorporan metodologías innovadoras. $Y$ esta tendencia requiere conocimientos y capacidades distintos, con mayor énfasis en el uso de la tecnología, en el trabajo en equipo, la comunicación, el liderazgo, la negociación o el sentido emprendedor, así como unas actitudes profesionales y personales diferentes.

En el entorno institucional se entiende como el capital humano compuesto de cualificación profesional con capacidades como la innovación, la creatividad, la adaptación al cambio, el sentido de pertenencia, etc. Gary Becker (1983) quien dio nombre en 1964 al término a través de su monografía Human Capital, y $\underline{\text { Schultz }}$ (1992), así como multitud de posteriores autores, defendían que el crecimiento económico para el mundo occidental dependía de su capital humano el cual debía poseer cualificación especializada, saber hacer y pericia como consecuencia del aprendizaje y de la experiencia.

En los entornos laborales, el término de competencia está directamente relacionado con la eficacia, eficiencia y flexibilidad de los trabajadores. Así, muchos expertos han llegado a afirmar que el término competencia ha suplantado al de cualificación como marcador de la categoría social con sus garantías y derechos. La educación basada en competencias busca responder a los requerimientos de la sociedad actual de la información o del conocimiento y qué mejor que hacerlo a través de las voces de los empleadores que son quienes gestionan las estimaciones de empleabilidad del mercado y por tanto a quienes hay que escuchar, desde esta investigación, para acercar el mundo laboral a las instituciones educativas, donde también el término competencia redunda tanto en formación docente como en reglada, ocupacional y continua.

\section{Las Competencias en la Universidad}

Según el Informe Hacia un Enfoque de la Educación en Competencias (Álvarez Morán, Pérez Collera y Suárez Álvarez, 2008) el concepto de competencia es amplio y abarca conocimientos, potencialidades, habilidades, destrezas, prácticas y acciones de diversa índole (personales, colectivas, afectivas, sociales, culturales) en los diferentes escenarios de aprendizaje y desempeño.

Una clasificación sencilla de las mismas sería esta:

- Competencias Básicas o Instrumentales, asociadas a conocimientos fundamentales que habitualmente se adquieren en la formación general y posibilitan el acceso al trabajo. Ejemplo: Nociones mínimas de lecto-escritura, comunicación oral, cálculo, lo que hoy podríamos denominar Competencias Clave.

- Competencias Genéricas o Transversales, relacionadas con los comportamientos y actitudes en el desempeño de las tareas de los distintos ámbitos de la producción o el servicio. Ejemplo: Capacidad para trabajar en equipo; saber planificar, habilidad para negociar, saber transmitir ideas, comunicar, liderar, delegar.

- Competencias Específicas o Técnicas o Especializadas, ligadas a la ocupación y no fácilmente transferibles a otros contextos laborales. Ejemplo: Operación de maquinarias especializadas, formulación de proyectos de infraestructuras concretas.

Las competencias laborales se componen por tanto de las actitudes, conocimientos y destrezas necesarias para cumplir exitosamente las actividades que conforman una función laboral, según estándares definidos por el sector productivo y teniendo en cuenta al individuo y al entorno. Las competencias laborales son acumulativas, es decir, para lograr un desempeño laboral óptimo es necesario contar de base con competencias básicas, que por lo general son previas a las competencias genéricas para más adelante llegar a poseer las competencias específicas. Por tanto, se podría decir que una competencia laboral es un constructo complejo que debe involucrar a las tres esferas, siendo las genéricas las que más cuesta encontrar, cosechar e implementar en las personas según la voz de los empleadores directivos de las principales compañías en España en las diferentes mesas redondas que se llevaron a cabo en el $6^{\circ}$ Congreso de Factor Humano organizado por IESE-IFAES (mayo, 2019), donde Manuel Pimentel, Consejero de Backer \& McKenzie, y ex Ministro de Trabajo, 
aludía a la necesidad de disponer de una estructura educativa y laboral que permita a las empresas competir en la búsqueda de talento al tiempo que den seguridad al empleado.

Las compañías consideran que existe una falta de competencias transversales competencias, las transversales (liderazgo, negociación, comunicación, delegación, gestión de conflictos, motivación y capacidad para motivar, etc.,) en las personas que buscan insertarse en el mercado laboral, considerando que entrenarlas es responsabilidad de las empresas cuando las personas están inmersas en el mercado laboral, pero que los entornos educativos deben potenciar más estas competencias durante los periodos formativos ya que se trata de un valor fundamental para las organizaciones y los mercados actuales.

Grossi (2012), en un estudio sobre la percepción de los estudiantes de ciencias sociales y jurídicas de la Universidad de Oviedo, mantiene que a las Instituciones de Enseñanza Superior se les demanda que generen y divulguen el conocimiento necesario para resolver los problemas de la sociedad actual, en beneficio de la transformación de estudiantes en ciudadanos capaces de desempeñar el rol que la sociedad del conocimiento les exige. Desde la crisis económica de finales de la década pasa$\mathrm{da}$, vivimos de cerca situaciones complejas que han afectado al modelo de estado de bienestar y consumo. En nuestros días, la crisis sanitaria por el Cov-19 apunta a un cambio perentorio y acelerado en el modelo de aprendizaje, en el entorno laboral y, en general, en el estilo de vida. De la Torre (2010) hablaba de urgencia en tomar conciencia sobre la importancia de no mantener modelos educativos ajenos a la realidad, que crean ciudadanos dependientes en lugar de pensadores libres y emprendedores, que favorecen la pasividad en lugar del pensamiento crítico y la creatividad. Esa urgencia no es cuestionable hoy día. En los entornos educativos domina un academicismo que se olvida, en muchas ocasiones, de la realidad la vida y de los problemas sociales y humanos, predomina el conocimiento teórico respecto al práctico y se ofrecen habitualmente enseñanzas descontextualizadas con la realidad y la vida.

La Sociedad en general, y el entorno laboral en particular, exigen a la Universidad que forme a las nuevas generaciones en competencias, actitudes y nuevos valores, como son la creatividad, la capacidad de resiliencia ante la adversidad, la capacidad crítica respecto a las informaciones recibidas, la convivencia y la solidaridad humana (De la Torre y Pujol, 2009).

Señala Grossi (2012) que entre las competencias de los estudiantes para integrarse a la realidad social y laboral, además de los conocimientos técnicos o estudios superados y posesión de titulación, se destacan las siguientes:

- Habilidad para la comunicación.

- Creatividad.

- Habilidades para la gestión del proyecto.

- Habilidad para el liderazgo.

- Habilidades para la gestión del cambio.

- Cultura básica empresarial.

- Capacidad para la identificación de problemas.

- Respeto a los valores y principios éticos universales y virtudes para la competencia.

La evolución de la sociedad estimula la competencia entre universidades por la búsqueda de un mercado potencial de clientes y usuarios, siendo aquellas que mejor se adapten al entorno actual quienes capten y desarrollen el mejor talento, contribuyendo así a la empleabilidad de personas capaces de saber y saber hacer. José Manuel Martínez (El País, 2017), profesor de Harvard, señala que no basta con ser experto en un solo campo, sino que el paso por la universidad debe suponer la obligación implícita de desempeñar diferentes roles, de ser transversal y multidisciplinar. Este profesor considera que el sistema educativo español invita a una formación "demasiado especializada" que no prepara para asumir retos desconocidos. Los empleos del futuro no están claros y por eso la especialización por sí sola ya no sirve. Hacen falta perfiles muy transversales para cubrir los nuevos puestos de trabajo que surgirán mañana y que aún hoy no conocemos. En Harvard, durante la primera semana lectiva de cada curso, se celebra la Shopping Week o semana de compras en la que cada profesor reseña en qué consiste su asignatura, la metodología de trabajo, los contenidos y la evaluación; los alumnos asisten a estas presentaciones y eligen las materias que conformarán su programa académico. Los docentes compiten por captar alumnos ya que no conseguir estudiantes puede impactar en su continuidad en la universidad. Este sistema empodera a los estudiantes y tiene la virtud de aportar capacidad de elección a éstos y a los profesores, quienes tienen libertad para modificar los contenidos, innovar con metodologías de aprendizaje hacia la mejora continua y liderar a través de una docencia inspiradora donde la conversación está muy presente durante todo el proceso de enseñanza-aprendizaje. El profesorado conoce las reglas del juego por adelantado y considera que este modelo aporta un grado de presión suficiente para ser positivo. Es para tomar nota.

En este contexto, uno de los retos presentes de la Universidad será crear entornos de aprendizaje que permitan desarrollar la autonomía del alumnado, que estimulen unos buenos resultados académicos a través del aprendizaje significativo, relevante y eficaz, que no olvide las competencias y habilidades que demanda un entorno laboral profesional y social cada vez más complejo; un aprendizaje que capacite para enfrentar las situaciones cotidianas laborales y sociales y motive en la ampliación conocimientos de manera continua y autónoma.

El IESE (2019), en un diagnóstico sobre las competencias profesionales necesarias para el empleo futuro, que recoge el punto de vista de las organizaciones, manifiesta que el $78 \%$ de las 53 empresas encuestadas afirman que el cambio comportamental de los clientes y/o usuarios, consecuencia de las nuevas demandas en base a sus nuevas necesidades, ha tenido un impacto altísimo que exige en las empresas empleados con capacidades distintas a las tradicionales para utilizar herramientas de mayor sofisticación, sobre todo a nivel tecnológico y en el uso de distintos idiomas, y para relacionarse en los entornos laborales con éxito a través del desarrollo de habilidades o competencias para el trabajo en equipo, para el aprendizaje continuo, el liderazgo o la dirección de personas, la comunicación, la innovación y creatividad, la toma de decisiones, la gestión de conflictos, la orientación a resultados, el compromiso, en definitiva, las competencias o habilidades que hoy demandan las organizaciones para mantenerse de forma competitiva en los actuales mercados locales, nacionales e internacionales.

El 52\% de las compañías considera, en términos generales, que el Sistema Educativo se orienta mucho más a conocimientos teóricos que prácticos, no restando importancia a los primeros, pero detectando grandes carencias en cuanto a capacidades importantes para la posterior vida laboral de las personas. Las empresas tienden a estructurarse de manera plana y transversal, con metodologías colaborativas, trabajo por proyectos y aplicación natural de modelos de Desing thinking (pensamiento de diseño) para fomentar la innovación que promueven la involucración de los empleados como protagonistas del cambio. Su aplicación permite que se generen mejores ideas y resultados en equipo. El 95\% de las empresas apunta a la resi- 
liencia y adaptabilidad como dos competencias prioritarias en las personas a contratar, sin embargo, más del 72\% manifiesta dificultades para encontrarlas. La inherente carencia de habilidades o competencias transversales en las personas impide que muchas empresas puedan evolucionar al ritmo necesario, despidiendo personas que son contratadas por sus aptitudes o capacidades en cuanto a conocimientos y son despedidas por sus actitudes o falta de competencias para el liderazgo. Señalan preferir personas con ganas y compromiso a personas con muchos saberes, puesto que manifiestan menor dificultad y menor coste a la hora de mejorar sus conocimientos que a la hora de desarrollar sus habilidades.

El informe, entre sus propuestas de mejora, señala que las empresas tienen por delante un gran papel para adaptarse al nuevo paradigma social. Uno de los puntos principales que se recogen como acción, se refiere a la mayor relación entre empresas y centros educativos, colaboración de las empresas en los planes educativos, presencia de profesorado universitario en organizaciones para conocerlas, sentir sus necesidades, comprender sus objetivos en el actual mercado para poder reforzar así el aprendizaje práctico orientado a la adquisición de competencias desde las aulas. Las empresas consideran que el acercamiento del profesorado a sus realidades serviría de palanca para promover un aprendizaje más práctico y más adaptado a lo que necesitan en los empleados presentes y futuros. Y viceversa, profesionales consolidados deberían impartir docencia en las universidades para acercar la realidad empresarial a los egresados y egresadas, compartir y generar espacios de pensamiento y trabajo con el personal docente, etc.

\section{Repensando modelos de competencias}

La enseñanza y el aprendizaje de las competencias y habilidades sociales requieren que desde todos los ámbitos se trabaje de forma coordinada y planificada: las universidades o instituciones de educación profesional, las empresas y los organismos públicos. Aunando el esfuerzo y la colaboración de todos, se puede lograr la responsabilidad colectiva de que cualquier persona esté capacitada para el trabajo y la sociedad futura, algo interesante como sociedad para el desarrollo de personas y organizaciones educativas, sociales y laborales más eficientes, sostenibles y responsables.

La sociedad y las organizaciones lo que demandan a la Universidad no son sólo conocimientos que versen sobre el aprendizaje de contenidos sino también que contribuya al desarrollo de las habilidades sociales, de las competencias de liderazgo, de inteligencia emocional, etc., claves para la posterior búsqueda y éxito en el empleo y en la vida de las personas, siendo la mejora del empleo a través de la optimización de las competencias de las personas la principal motivación para realizar esta investigación.

Modelo de estudiante-empleado T

La traumática recesión de los mercados internacionales en el año 2008 desembocó en una crisis que afectó a los trabajadores y donde la educación experimentó crecientes críticas a causa de las limitadas oportunidades que ocasionaba. Nace así el nuevo enfoque en forma de $\mathrm{T}$, con un enfoque educativo integral sobre el estudiante-empleado en el que las personas incorporan paulatinamente conocimientos mientras entrenan y conquistan habilidades y valores que les dotan de garantías de futuro en términos económicos, laborales, sociales y culturales (Arreguit y Hugues, 2019).

Este enfoque defiende y representa a estudiantes y empleados innovadores con conocimientos técnicos especializados y profundos en una materia o en su disciplina que además tienen aptitudes y habilidades, válidas en cualquier entorno laboral o categoría, como la capacidad de comunicación, el liderazgo, la perseverancia, el trabajo en equipo, la empatía, la gestión del cambio, la resolución de conflictos, la negociación, etc., que les convierten en poseedores de perfiles talentosos.

Sostiene que el gran bloque de la T, la parte vertical, está integrado por la profundidad de conocimiento y la capacidad para ponerlo en funcionamiento a través de las habilidades profesionales. El modelo T provoca una conversación acerca del grado en el que los enfoques innovadores de diseño de metodologías de aprendizaje tienen en cuenta alternativas para formar y alinear el talento entre la experiencia la educación superior y las oportunidades profesionales posteriores. Sobre la T reposa el entusiasmo por un nuevo modelo de currículo y de aprendizaje, pero este modelo no significa sencillamente agregar habilidades y competencias a los planes de estudios, sino que requiere de la integración y el uso innovador e intencional de la tecnología, los espacios y los contextos para diseñar experiencias de aprendizaje dotadoras de conocimientos y de saber hacer mientras se conforma el saber ser pudiendo así hacer una crónica del desarrollo de un alumno en una $\mathrm{T}$.

El sombrero horizontal lo conforman los resultados, los logros y los éxitos cuyos pilares son la comprensión superior y la habilidad de comunicarse y trabajar en equipos multiculturales y globales donde la visión y el liderazgo se convierten en cualidades y factores determinantes.

El modelo T ilustra cómo los componentes críticos del aprendizaje, los conocimientos académicos, junto con la comprensión y mejora de uno mismo y con los demás, facilitan el éxito de las personas en su carrera laboral y, por ende, en su vida.

Con la implementación de este modelo desde la educación superior se pretende contribuir a la generación de patrones de comportamiento que requieren de un pensamiento abierto, innovador y flexible; el autoaprendizaje, autoconocimiento y la actitud se consolidan como las características que definen los límites de uno mismo.

Las instituciones educativas, para adaptarse al modelo $\mathrm{T}$, necesitan desarrollarse creativamente y promover de manera intencional acciones interdisciplinarias en sus entornos que permitan a los estudiantes comprender perspectivas alternativas, adquirir conocimientos y experimentar las competencias mediante el trabajo en equipo para la resolución de problemas a través de experiencias de aprendizaje coherentes dentro y, probablemente lo más importante, mediante la participación en y/o con entornos fuera de las aulas universitarias (Griffiths y Guile, 2003).

Así, una persona que se forma y desarrolla bajo este modelo durante su etapa universitaria, posee la capacidad construir relaciones sostenibles y comprender motivos, roles y responsabilidades, y adquiere la conciencia de que el componente más crítico (de la T) es el conocimiento individual de uno mismo, el autonocimiento; la comprensión del Yo es fundamental para desarrollar la capacidad de funcionar con propósito, confianza y conciencia y avanzar de forma profesional acorde a los valores y las motivaciones que, al conocerlas, se siguen mejorando así como el resto de competencias; la consciencia global del entorno, el conocimiento y las habilidades conforman, bajo este modelo, al individuo exitoso. William Damon (2009) dice que se trata de enfatizar, con la aplicación de este modelo de estudiante, en la importancia del propósito en todos los aspectos de la juventud distinguiendo el objetivo de una meta personalmente significativa al compromiso del logro socialmente beneficioso extendiéndose más allá de uno mismo y consecuente para el mundo.

La fuerza del modelo $\mathrm{T}$ para conseguir este propósito se basa en tres principios clave para el profesorado: 
- Ser innovador, tener apertura de mente hacia formas creativas para estimular el aprendizaje a través del plan de estudios pero empleando los recursos tecnológicos (Friedman, 2016).

- Ser intencional, dirigir el aprendizaje hacia la adquisición de contenidos y el desarrollo de las competencias y de la personalidad (Dormehl, 2016).

- Ser integrador, practicar la reflexión, las conversaciones, la experiencia propia para ayudar al alumnado a visualizar su propio crecimiento (Dawson, 2013).

La enseñanza-aprendizaje a través de la T requiere de prácticas reales que aseguren a los estudiantes la comprensión de cómo pueden desarrollar sus habilidades para convertirse en T-profesionales, del estudiante $\mathrm{T}$ al empleado T. Es, en definitiva, un modelo que incorpora cambios con el objeto de que cada persona consiga lo mejor de sí misma desde la realidad de sus competencias y con la consciencia y la capacidad para el autoaprendizaje que le permitan mejorar de manera continua y adaptarse al entorno y al futuro profesional con éxito, manando de aquí o siendo esto requisito indispensable para el desarrollo de las personas en la sociedad y conociendo, como hemos reflejado con anterioridad, los devastadores efectos de la falta de empleabilidad. El modelo manifiesta que, si las competencias personales y profesionales marcan de manera trascendental nuestra vida, estando ubicadas aquellas afines al liderazgo en la parte superior de la barra horizontal de la T, que podemos entender como metacompetencias y las hemos venido definiendo en diversas ocasiones, será sin duda óptimo comenzar a trabajarlas en los sistemas educativos y sobre manera en las instituciones de nivel superior como la universidad.

La Consultora Deloitte manifiesta que en cinco años vista este será el perfil más demandado en las empresas y en las personas estará el eje central de las compañías, las cuales, dejando a un lado la preocupación de los últimos tiempos por la eficiencia organizativa (lean), el cambio cultural y control económico, optarán por poner el foco en el empleado optando por el perfil tipo $\mathrm{T}$ como dominante en el futuro según el 72 \% de los CEOs encuestados en el estudio (Deloitte, 2018).

El modelo T combina ambas (Heikkinen, 2018), competencias y conocimientos, reconociendo a aquellos profesionales portadores de la $\mathrm{T}$ considerándolos como portadores de las habilidades necesarias para cruzar los límites que favorecen el éxito profesional y ponen en valor el talento. La educación superior, la educación universitaria, debe por tanto promover un modelo potencial para aprender las habilidades de cruce de límites, lo que resultaría comenzar a sentar la base de la T. Este autor ha realizado numerosos estudios, como el "Studying the Aspects of Knowledge Creation in the LAB Studio Model", donde incluye hallazgos sobre cómo comúnmente se utiliza la educación basada en el estudio en todo el mundo en la educación superior sin apenas cruce activo de límites internos y externos entre los estudiantes, proponiendo para la Universidad de Finlandia una disertación que se base en este modelo y contribuya a la colaboración universidad-empresa a través de nuevas configuraciones de aprendizaje interdisciplinar de los trabajadores del conocimiento.

\section{Modelo de estudiante-empleado OTA}

Arreguit y Hugues (2019) consideran, del estudio exhaustivo del modelo $\mathrm{T}$, un segundo enfoque que lo amplía y lo mejora buscando el ideal del empleado competente, mediante las voces de los empleadores, capaz de desempeñar un trabajo en el entorno actual y futuro. Dilata, este modelo OTA, el concepto de estudiante tipo $\mathrm{T}$ manteniendo el significado de la $\mathrm{T}$, pero dando un paso más y situando en la $\mathrm{O}$ el pensamiento y la visión global, y en la A la acción, el emprendimiento, la actuación consciente, el hacer.

El estudiante-empleado tipo OTA, a través de la consciencia de su lugar dentro del sistema, de sus relaciones, de sus vínculos y posiciones, de sus dependencias, de sus conflictos y de la complejidad en general del entorno, es capaz de comprender y resolver los principales problemas y desafíos, influyendo con sus decisiones en el logro de su propia eficiencia, en la consecución de sus objetivos en función de las posibilidades, en la definición de sus responsabilidades. Este modelo defiende que el individuo debe tener conocimiento y experiencia en más de una disciplina y comprender otras, así como ser comprendido por especialistas en ellas. Esto requiere de capacidad de adaptación a distintos contextos profesionales, técnicos o socioculturales, así como de rapidez para adquirir nuevos conocimientos y comprender múltiples áreas o especialidades; de aquí, entre otras, la importancia de la formación continua en los entornos laborales.

Este enfoque recoge la necesidad de estar dispuesto a actuar y poder hacerlo, los conocimientos multidisciplinares son imperiosos, pero no suficientes, es necesario contar con la experiencia para actuar, para avanzar, para cambiar y evolucionar a través de la puesta en marcha de metaprocesos, procesos que utilizamos para más de una situación o tarea y que aluden a la actitud. Las personas, para evolucionar o conseguir el máximo potencial de uno mismo precisan de actitud, de actuación, de la A.

En términos empresariales, un empleado tipo OTA sería aquella persona con conocimientos sólidos, con habilidades y competencias para desempeñarse profesionalmente en el actual entorno laboral, aportando valor y competitividad, que disponga de actitud hacia el liderazgo, sepa trabajar en equipo, sea capaz de gestionar equipos de trabajo, se desenvuelva en entornos digitales en continua transformación, comprenda los nuevos comportamientos del mercado y de los clientes, y en definitiva tenga el perfil competencial que las empresas vienen demandando.

\section{Conclusiones y discusión}

La revisión documental que se ha realizado en este artículo pretende valorar un justificado llamamiento a la universidad acerca del carácter estratégico e innovador de la implementación paulatina del modelo en sus estructuras, adaptándose a las demandas de la sociedad como marco global en el que se desarrolla y al que, en cierta medida, sirve o aporta un servicio desde un punto de vista mayormente empresarial.

El escrutinio documental en torno a los modelos presentados y sus resultados en otros países, lleva a pensar en su implementación en la Universidad de Oviedo en beneficio del talento de la región, una masa salarial competente y capacitada, que cubra la demanda de los puestos actuales en los diferentes ámbitos y ponga en marcha nuevas ideas de negocio que, sin duda, pueden estar incentivadas por la posibilidad de deslocalización de muchos puestos de trabajo, la adquisición de visión para emprender y la exportación de productos y servicios, entre otros (Álvarez-Arregui, 2019). Siendo conscientes de que la innovación afecta a todas las esferas de la vida, puede ser necesaria e inminente la puesta en marcha de nuevos enfoques o modelos en el sistema educativo; si desaparecen empleos tradicionales como es sabido, y aparecen ocupaciones nuevas que requieren nuevas competencias, las universidades deberían adaptarse y cambiar sus planes de estudios manteniendo su prestigio como institución de conocimiento superior, introduciendo nuevos conocimientos y experiencias, aunque deba ser en detrimento de algunas asignaturas tradicionales. 
El nuevo conocimiento y la experiencia no solo ocurren en las universidades (Arreguit y Hugues, 2019), también en la industria y en los organismos públicos y sociales; todos deben involucrarse bajo un enfoque real de educación dual y continuada que permita ir al ritmo de la innovación, es necesario engranar estas esferas para afrontar los trabajos y los retos presentes y futuros. Una investigación adicional sería estudiar las competencias que el entorno laboral en Asturias desea para los futuros empleados y analizar estos resultados en base modelo de estudiante/empleado tipo OTA para obtener datos sostenibles acerca de la importancia de implantar acciones en la Universidad sustentadas bajo este modelo y, por tanto, catalizadoras del talento demandado por los mercados.

También las empresas y las organizaciones tienen que propiciar cambios en sus estructuras para mantenerse en el mercado, pero, para ello, aluden continuamente a la necesidad de contratar personas preparadas, apuntan a la falta de talento, a las dificultades para encontrar empleados con habilidades blan- das, con liderazgo, con competencias no sólo instrumentales sino también genéricas y específicas (Jericó, 2008). Este es el motivo principal del incremento creciente de planes de formación en las empresas destinados a la mejora de las habilidades directivas y de trabajo en equipo.

La capacidad de adquirir nuevos conocimientos será más valiosa que el propio conocimiento en el futuro, quizás porque es tiempo de desaprender lo aprendido para volver a aprender. Quedarse a la espera sin intervenir ya no es una opción; siendo conscientes de que el cambio necesario es sobre todo cultural, con lo que esto conlleva, y compartiendo visión con Álvarez-Arregui y Arreguit (2019) y Arreguit y Hugues (2019), se propone profundizar en las posibilidades del modelo OTA en el entorno universitario, tal y como se viene haciendo desde el Instituto de Investigación e Innovación Educativa (INIE) de la Universidad de Oviedo por considerarlo capaz de mejorar a las personas para el empleo del futuro. 


\title{
Society, University, Business and Talent in times of change. Learn and unlearn to start again
}

\author{
Vanessa Argüelles Rodríguez \\ vanessaarguellesapfsgrupo.com \\ University of Oviedo - PFSGroup (Spain) \\ ORCID: https://orcid.org/0000-0003-3761-5386
}

\begin{abstract}
University, Business and Society have been keeping a close relationship conditioned by the economic, social, political and functional elements that operate in each historical moment. Today, the academic culture and a functionalist vision of the acquisition of leadership skills and competences seek to coexist and engage in university environments. This article, prepared on the basis of a documentary review, analyses the concept of talent from the companies perspective, and studies the change towards models of students who favour employees more prepared to work, with aspirations and protagonists of their own learning to have more opportunities that improve their employability and, therefore, their quality of life. This research invites reflection on whether universities should take a new approach to adapt to an emerging context and to foresee future situations that maintain them as agents and educational benchmarks.
\end{abstract}

Keywords: learning, teaching, training, talent, competence and leadership. 


\section{Introduction}

In the current capitalist society, the employability rates in Spain continuously affect all areas of our lives and, according to the Centre for Sociological Research (Centro de Investigación Sociológicas, CIS, 2020), the Spanish population is increasingly concerned about the economic sphere, the unemployment figures and the precariousness of jobs. Unemployment remains as the main problem for more and more citizens, and this increasing concern coincides with the publication in early February 2020 of data indicating that the month of January 2020 had closed with more than 90,000 new unemployed registrations in public employment offices, the worst start to the year since 2013. All this raises a constant question in society and in the institutions, that guides in part this study on the new competences needed to meet the current demand for labour.

Society, employment and education are conceived as spheres that have been interconnected throughout history but face a changing, interrelated, technological and global scenario to which they must adapt in order to remain sustainably. There is a constant reference to the need to count on talented people, to the importance of the linkage between the educational system and the productive system, to the role of the University as a guarantee for that talent (Álvarez-Arregui \& Arreguit, 2019). This is how the first evidences that respond to the objectives of this research emerge: to know what is what labour organizations understand by talent, to catalogue the skills that the labour market currently demands, to know the importance of labour stability for the systems and especially for people, and finally, to propose a university model in accordance with the new scenario.

\section{A conceptual approach for Talent}

In order to reach objective and valid conclusions, we need to define what is understood by Talent from the perspective or approach considered in this desk-based research, namely the work environment, in order to materialize it in those key skills and abilities for its development and identified, and related to knowledge, know-how, wanting to do and being. Since there is no consensus in the academic literature regarding the term, each organization delimits what it considers a talented profile according to its current or future needs (Tansley, 2011). According to the definition that Jericó, Botín and Medina (2008) have given to the concept of talent, we understand it as the greatest asset of organizations and therefore as the factor that will make the strategic difference between excellent and ordinary companies. Their research also remarks the fact that, being one of the most valuable resources, talent is often the worst managed asset in working environments, but this would be the result of further research which is not the focus of this study.

There have been numerous studies that have used the term talent defined as certain outstanding skills in a multitude of sciences such as biology, psychology, pedagogy, sociology, philosophy and business. This study analyses talent from the point of view of the demand that organizations are making of it (Lorenzo, 2006). Likewise, talent has been related in numerous occasions and in a wrong or confused way with concepts such as genius, intellectual giftedness or even excellent achievement, attributing it to individuals that have stood out in some subject or science. Thus, Berguer (1997) named in this way the individuals who obtained a high level of intelligence as a result of a test of subjects with general talent; Sisk (1993) had referred to a more determined talent that had to do with individuals who excelled in performance on tests in more specific aptitudes such as capacity or ability for leadership, understanding leaders as people who use certain skills, who possess high levels of negotiation, have and demonstrate self-confidence, responsibility, dominant tendency and susceptibility to adapt to new situations.

In the field of employment, according to Jericó (2008) the concept of talent focuses on the set of skills related to four meanings, three of which correspond to work, defining talent as the ability to obtain remarkable results in the exercise of intelligence, aptitudes and skills. Talent is therefore synonymous with aptitude, capacity, skills and suitability. From this point of view, to possess talent would be to be well endowed for, to be capable to, to have brilliant qualities for, to be fit for, to be useful for. And here is where skills come into play, because we understand that they are the ones that serve as indicators to unravel in actions what is understood as talent.

Baigorri (2018) states that talent is based on certain personal conditions (partially inherited) that will be projected into various fields of our activity, being very important to understand that talent does not develop spontaneously or randomly and not always at an early age, but it is the result of applying personal effort, will and the development of what initially are nothing more than dubious potentialities or skills. Talent will expand to the maximum when favourable circumstances arise for it at the family, personal, educational, social and professional levels. Talent cannot be developed if it is not cultivated, and for this development, the first requirement is to identify it (Touron, 2015).

Although neither in business practice nor in academic literature there is a consensus on the definition of talent, it is possible to state that, in general terms, both areas refer to the same type of skills profile, regardless of the specific name given to it (Hernández Lechuga, 2017). The division, therefore, is always based on skills that generate excellent results. It would be necessary to clarify the meaning of the term from the point of view of labour organizations, which is the one taken to carry out this research.

\section{Competences and skills}

The term skills was first introduced in the 1920s in the United States, but it was during the labour boom of the 1960s and 1970s when it emerged to stay thanks to David Mc Clelland (1973) and is taken up again strongly in the 1990s with Goleman's (1996) formulations about emotional intelligence.

Short (1984) stated different points of view to define and understand the skills:

- As conducts, behaviours or acts that result in a specific performance.

- As a set of specific knowledge and abilities that must be put in place to carry out certain actions.

- As the capacity added to the abilities, behaviours and knowledge of the individual. It is a third party who values a person's competence, does not meet defined but changing standards.

- As a quality or state of being, something holistic where behaviours, knowledge, abilities and intentions are derived from the state of being.

To understand the concept of skills, we find among academics many definitions that do not provide a practical interpretation necessary for companies (Mertens, 1998); however, the term is used in the areas of HR and affecting employed and future personnel. 
Skills, basically, are in the eyes of the labour market the professional answers that an individual gives to the requirements of his or her job (understanding a job as that which has a concrete location, a specific activity, a sector, a socio-political and economic environment, etc.) (Pérez Escoda \& Sabariego Puig 2015).

In order to respond to all these indicators or requirements, the skills have been changing and evolving, undergoing various conceptualizations; they commenced as people's abilities to perform a certain activity or function in a job (more task-centred approaches); then they became traits and characteristics that excellent professionals had, that led to brilliant results in their respective organizations (theories more focused on the professional profile), to later be discussed the key skills to reach excellence. Finally, there has been a trend towards models that understand skills in a holistic and complex manner where the task, the professional excellence, the personal training and development and the skills and/or attitude to adapt to the working environment with changing and globalized markets come into play.

In January 2019, IESE Business School (IESE), in its report "El futuro del empleo y las competencias profesionales del futuro: la perspectiva de las empresas" (The future of employment and the professional skills of the future: the perspective of companies), whose backbone is the great challenge of alleviating the unemployment rate in Spain, analyses the skills required by 53 leading companies in the country -employing almost half a million people in Spain and almost three million worldwide in December 2017-, to cover their needs. The report specifies that almost three out of four companies find difficulties in filling the jobs offered, both in terms of knowledge in some areas and in terms of professional skills and attitudes. Specifically, the companies interviewed showed great concern about the change in professional skills that automation and the digital revolution are generating (72\% of these companies consider that the digital revolution has a high or very high impact on the change of required profiles and $56 \%$ of these companies find that the impact of automation is high or very high) while highlighting a shortage of leadership competences, skills related to attitude, effort, commitment, change management, social and teamwork skills, stress management and goal orientation, the so-called soft skills in business environments.

The report states that the trend in companies is the evolution towards flatter and more transversal structures, with different ways of working, which incorporate innovative methodologies. And this trend requires different knowledge and skills, with a greater importance to be given to the use of technology, the teamwork, the communication, the leadership, the negotiation or the entrepreneurship, as well as different professional and personal attitudes.

In the institutional environment, it is understood as human capital composed of professional qualifications with capabilities such as innovation, creativity, adaptation to change, sense of belonging, etc. Gary Becker (1983), who gave the term its name in 1964 through his monograph Human Capital, and Schultz (1992), as well as many subsequent authors, maintained that economic growth for the western world depended on its human capital, which should possess specialized qualifications, know-how and expertise as a result of learning and experience.

In working environments, the term skills are directly related to the effectiveness, efficiency and flexibility of workers. Thus, many experts have even gone so far as to state that the term skill has replaced that of qualification as a marker of social category with its guarantees and rights. Skills-based education seeks to respond to the requirements of today's information or knowledge society, and the most direct way to do so is through the voices of employers, who are those who manage the market employability estimates and therefore those who must be listened to, from this research, in order to bring the world of work closer to educational institutions, where the term skills is also used to refer to teacher training as well as to formal, occupational and continuous training.

\section{Skills at University}

According to the report 'Hacia un Enfoque de la Educación en Competencias' Towards an Approach to Education in Skills (Álvarez Morán, Pérez Collera \& Suárez Álvarez, 2008) the concept of skills is broad and encompasses knowledge, potentials, abilities, practices and actions of various kinds (personal, collective, affective, social, cultural) in different learning and performance scenarios.

A simple classification of skills would be the following:

- Basic or Instrumental Skills: linked to fundamental knowledge usually acquired in general training and enable access to work. Example: Minimum notions of reading and writing, oral communication, calculation, what today we could call Key Skills.

- Generic or Transversal Skills: related to behaviours and attitudes in the performance of tasks in the different areas of production or service. Example: Capacity to work in a team; know how to plan, ability to negotiate, know how to transmit ideas, communicate, lead, delegate.

- Specific or Technical or Specialized Skills: linked to the occupation and not easily transferable to other work contexts. Example: Operation of specialized machinery, formulation of specific infrastructure projects.

Labour skills are therefore composed of the attitudes, knowledge and capabilities necessary to successfully carry out the activities that make up a work function, according to standards defined by the productive sector and taking into account the individual and the environment. Labour skills are cumulative, that is, in order to achieve an optimum work performance, it is necessary to have basic skills, which generally precede generic skills in order to later possess specific skills. Therefore, it could be said that a labour skill is a complex construct that must involve all three spheres, being the generic ones those that are most difficult to find, harvest and implement in people according to the employers of the main companies in Spain, which took part in the different round tables held within the framework of the $6^{\text {th }}$ Human Factor Congress organized by IESE-IFAES (May, 2019), where Manuel Pimentel, Director of Backer \& McKenzie, and former Minister of Employment of the Government of Spain, remarked the need for an educational and employment structure that allows companies to compete in the search for talent while providing security for the employee.

Companies consider that there is a lack of transversal skills (leadership, negotiation, communication, delegation, conflict management, motivation and capacity to motivate, etc.,) in people seeking to enter the labour market, considering that exercising them is the responsibility of companies when people are immersed in the labour market, but that educational environments should further enhance these skills during training periods since they provide a fundamental value for today's organisations and markets.

Grossi (2012), in a study on the perception of social and legal science students at the University of Oviedo, maintains that Higher Education Institutions are required to generate and dis- 
seminate the knowledge needed to solve the problems of today's society, to benefit the transformation of students into citizens capable of playing the role that the knowledge society requires for them. Since the economic crisis at the end of the last decade, we have closely experienced complex situations that have affected the welfare and consumption state model. Nowadays, the health crisis derived from the COVID-19 pandemic points to a peremptory and accelerated change in the learning model, in the working environment and, in general, in our lifestyle. De la Torre (2010) remarked the urgency of becoming aware of the importance of not maintaining educational models divorced from reality, which create dependent citizens instead of free thinkers and entrepreneurs, who favour passivity instead of critical thinking and creativity. That urgency is not questionable today. In educational environments, an academicism that often forgets reality and social and human problems dominates; theoretical knowledge predominates over practical knowledge; and teachings are usually offered out of reality and life contexts.

Society in general, and the working environment in particular, require the University to train new generations in skills, attitudes and new values, such as creativity, resilience capacity in the face of adversity, critical capacity regarding the information received, coexistence and human solidarity (De la Torre \& Pujol, 2009).

Grossi (2012) states that, among the skills essential for the students to integrate themselves into the social and labour reality, in addition to technical knowledge or studies passed and possession of a degree, the following stand out:

- Communication skills.

- Creativity.

- Project management skills.

- Leadership skills.

- Change management skills.

- Basic business culture.

- Capacity to identify problems.

- Respect for universal values and ethical principles and virtues for competition.

The evolution of society stimulates competition between universities in the search for a potential market of clients and users, being the ones that best adapt to the current environment those who capture and develop the best talent, thus contributing to the employability of people capable of knowing and knowing how to do. José Manuel Martínez (El País, 2017), professor at Harvard, points out that it is not enough to be an expert in a single field but the time spent at university must also involve the implicit obligation to play different roles, to be transversal and multidisciplinary. For this professor, the Spanish educational system invites to a "too specialised" training that does not prepare students to take on unknown challenges. The jobs of the future are unclear and therefore specialization alone no longer works. Very transversal profiles are needed to cover the new jobs that will arise tomorrow and that we still do not know today. At Harvard, during the first teaching week of each course, the Shopping Week is held, in which each professor explains what their subject consists of, the work methodology, the contents and the evaluation; thus, the students attend these presentations and choose the subjects that will compose their academic program. Teachers compete to attract students since not getting students can impact their continuity in the university. This system empowers students and has the virtue of providing them with the ability to choose, which is also an option for the teachers, who are free to modify the contents, innovate with learning methodologies towards continuous improvement, and lead through inspiring teaching where the conversation is very present throughout the teaching-learning process. Teachers know the rules of the game in advance and consider that this model brings a sufficient degree of pressure to be positive. It is worth reflecting on this.

Within this context, one of the current challenges of the University will be to create learning environments that allow the development of the autonomy of the students, that stimulate good academic results through meaningful, relevant and effective learning, that does not forget the skills and abilities that an increasingly complex professional and social work environment demands; a learning that trains how to face the daily work and social situations, and stimulates the expansion of knowledge in a continuous and autonomous way.

According to an IESE (2019) diagnosis on the professional skills needed for future employment, which includes the point of view of employers, $78 \%$ of the 53 companies surveyed find that the behavioural change of customers and/or users resulting from new demands based on their new needs, has had a very high impact in the company. This impact requires enterprises to hire employees with skills other than traditional ones to be able to use more sophisticated tools, especially at the technological level and in the use of different languages, and to successfully interact in working environments through the development of skills or competences for teamwork, continuous learning, leadership or management of people, communication, innovation and creativity, decision making, conflict management, results orientation, and commitment. In short, these are the skills that organizations demand nowadays to remain competitive in today's local, national and international markets.

In general terms, $52 \%$ of companies consider that the Educational System is much more oriented to theoretical knowledge than to practice, without downplaying the importance of the former but detecting great deficiencies in terms of important practical skills for the subsequent working life of the students. Companies tend to be structured in a flat and transversal way, using collaborative methodologies, project work and natural application of Design Thinking models to foster innovation, which promote the involvement of employees as central actors of the change. Its application stimulates better ideas and results generated in teams. $95 \%$ of companies remark resilience and adaptability as two priority skills in people to be hired; however, more than $72 \%$ highlight difficulties in finding them. The inherent lack of transversal skills in people prevents many companies from evolving at the necessary pace, dismissing people who are hired because of their skills or abilities in terms of knowledge and are fired due to their attitudes or lack of leadership skills. They declare to prefer people with want and commitment to people with a lot of knowledge, since they find less difficulty and less cost when it comes to improving knowledge than when developing skills.

As proposals for improvement, the report states that companies have a great role to play in adapting themselves to the new social paradigm. One of the main points included as an action, refers to the greater relationship between companies and educational centres, to the collaboration of companies in educational plans, to the presence of university professors in organizations to get to know them, feel their needs and understand their objectives in the current market in order to strengthen practical learning oriented to the acquisition of skills from the classroom. Companies consider that bringing teachers closer to their realities would serve as a lever to promote a more practical learning, more adapted to what they need in present and future employees. And vice versa, consolidated professionals should teach 
at universities to bring the business reality closer to graduates, share and generate spaces for thought and work with the teaching staff, etc.

\section{Rethinking the skills models}

The teaching and learning of social skills require that all the areas involved work in a coordinated and planned way: universities or vocational education institutions, companies and public bodies. By combining the efforts and collaboration of all of them, it is possible to achieve the collective responsibility that any person is qualified for the future work and society, something interesting as a society for the development of more efficient, sustainable and responsible people and educational, social and labour organizations.

Society and organizations demand from the University not only knowledge about learning content but also to contribute to the development of social skills, leadership skills, emotional intelligence, etc., keys for the subsequent search for and success in employment and in people's lives. The improvement of employment through the optimization of people's skills is the main motivation to carry out this research.

\section{Student-Employee Model (T model)}

The traumatic recession in international markets in 2008 led to a crisis that affected workers and where education and training came under increasing criticism because of the limited opportunities it was providing. This is how the new T-shaped approach was born, including a comprehensive educational approach on the student-employee, in which people gradually incorporate knowledge while training and conquering skills and values that provide them with guarantees for the future in economic, labour, social and cultural terms (Arreguit \& Hugues, 2019).

This approach advocates and represents innovative students and employees with specialized and in-depth technical knowledge in a specific subject or in their discipline, who also have skills and abilities that are valid in any work environment or category, such as communication skills, leadership, perseverance, teamwork, empathy, change management, conflict resolution, negotiation, etc., that make them talented profiles.

It also maintains that the big block of the $\mathrm{T}$, the vertical part, is integrated by the depth of the knowledge and the ability to put it to work through professional skills. The T model provokes a conversation about the degree to which innovative approaches to the design of learning methodologies consider alternatives for training and aligning talent between higher education experience and subsequent career opportunities. On the T rests the enthusiasm for a new model of curriculum and learning; however, this model does not simply mean adding skills to the curriculum, but requires the integration and innovative and intentional use of technology, spaces and contexts, in order to design learning experiences that endow knowledge and know-how while knowing how to be is formed, thus being able to chronicle the development of a student in a $\mathrm{T}$.

The horizontal part of the $\mathrm{T}$ is made up of results, achievements and successes whose pillars are superior understanding and the ability to communicate and work in multicultural and global teams, where vision and leadership become virtues and determining factors.

Model T illustrates how the critical components of learning, the academic knowledge, together with understanding and improving oneself and the others, facilitate the success of people in their work careers and, therefore, in their lives.
The implementation of this model from higher education is intended to contribute to the generation of behaviour patterns that require open, innovative and flexible thinking; self-learning, self-knowledge and attitude are consolidated as the characteristics that define the limits of oneself.

Educational institutions, in order to adapt themselves to the $\mathrm{T}$ model, need to creatively develop and intentionally promote interdisciplinary actions in their environments that enable students to understand alternative perspectives, acquire knowledge and experience skills through teamwork for problem solving through coherent learning experiences within, and, probably most importantly, through participation in and/or with environments outside university classrooms (Griffiths \& Guile, 2003).

Thus, a person who is trained and developed under this model during his or her university stage, has the capacity to build sustainable relationships and understand reasons, roles and responsibilities, and becomes aware that the most critical component (of the $\mathrm{T}$ ) is the individual knowledge of oneself, the self-knowledge; the understanding of oneself is fundamental to develop the capacity to act with purpose, confidence and awareness and to advance professionally according to the values and motivations that, when knowing them, continue to improve as well as the rest of the skills; the global awareness of the environment, knowledge and skills make up, under this model, the successful individual. William Damon (2009) declares that the application of this model of student tries to emphasize the importance of the purpose in all the aspects of the youth distinguishing the objective of a goal personally significant, from the commitment of the socially beneficial achievement, extended beyond oneself and consequent for the world.

The strength of the T model to achieve this purpose is based on three key principles for professors:

- To be innovative, to have an open mind towards creative ways to stimulate learning through the curriculum but using technological resources (Friedman, 2016).

- To be intentional, to direct the learning towards the acquisition of content and the development of skills and personality (Dormehl, 2016).

- To be an integrator, to practice reflection, conversations and own experience to help students visualize their own growth (Dawson, 2013).

Teaching-learning through the T model requires real practices that ensure students understand how they can develop their skills to become T-professionals, from T-student to T-employee. It is, in short, a model that incorporates changes in order for each person to get the best of themselves from the reality of their skills and with the awareness and capacity for self-learning that will allow them to improve continuously and adapt to the environment and to the professional future with success, arising from here or being an indispensable requirement for the development of people in society, and knowing, as we have previously reflected, the devastating effects of the lack of employability. The model states that if personal and professional competences mark our lives in a transcendental way, being located those related to leadership in the upper part of the horizontal bar of the $T$, which we can understand as meta-competences and we have been defining them in several occasions, it will be undoubtedly optimal to start working them in the educational systems and in a higher level institution such as the university.

The consultancy firm Deloitte states that in five years, this will be the most demanded profile in companies and in people, being the central axis of the enterprises. Leaving aside the con- 
cern of recent times for organizational efficiency (lean), cultural change and economic control, they will choose to put the focus on the employee by opting for the T-type profile as dominant in the future, according to $72 \%$ of CEOs surveyed in the study.

The $\mathrm{T}$ model combines both (Heikkinen, 2018), skills and knowledge, recognizing $\mathrm{T}$-professionals considering them as owners of the necessary skills to cross the limits that favour professional success and value talent. Higher education, university education, must therefore promote a potential model for learning the skills of crossing boundaries, which would result in beginning to lay the foundation of the T. This author has carried out numerous studies, such as the "Studying the Aspects of Knowledge Creation in the LAB Studio Model", where he includes findings on how study-based education is commonly used throughout the world in higher education with hardly any active crossing of internal and external boundaries between students, proposing a dissertation for the University of Finland based on the T model, which will contribute to university-industry collaboration through new configurations of interdisciplinary learning by knowledge workers.

\section{Student-Employee Model (OTA Model)}

Arreguit \& Hugues (2019) consider, from the exhaustive study of the T model, a second approach that broadens and improves it by seeking the ideal of the skilled employee through the voices of employers, able to perform a job in the current and future environment. This OTA model dilates the concept of the T-type student, keeping the meaning of the T but taking a further step and placing the global thinking and vision in the $\mathrm{O}$, and the action, the entrepreneurship, the conscious action, and the doing, in the A.

The OTA-type student-employee, through their awareness of their place within the system, of their relationships, of their links and positions, of their dependencies, of their conflicts and of the complexity of the environment in general, is able to understand and solve the main problems and challenges, influencing with their decisions in the achievement of their own efficiency, in the achievement of their objectives according to the possibilities, in the definition of their responsibilities. This model defends that the individual must have knowledge and experience in more than one discipline and understand others as well as be understood by specialists in them. This requires the ability to adapt to different professional, technical or socio-cultural contexts, as well as rapidity in acquiring new knowledge and understanding multiple areas or specialties; hence, among others, the importance of continuous training in work environments.

This approach reflects the need to be willing to act and be able to do it; multidisciplinary knowledge is imperative, but not sufficient, it is necessary to have the experience to be able to act, to move forward, to change and evolve through the implementation of meta-processes, processes that we use for more than one situation or task and that refer to the attitude. In order to evolve or achieve one's maximum potential, people need attitude, action, the A.

In business terms, an OTA-type employee would be a person with solid knowledge, with skills and competences to perform professionally in the current work environment, providing value and competitiveness, who has an attitude towards leadership, knows how to work in a team, is capable of managing work teams, develops in digital environments in continuous transformation, understands the new market and customer behaviours, and in short has the competency profile that companies have been demanding

\section{Conclusions and discussion}

The documentary review that has been carried out in this article aims to assess a justified call to the university about the strategic and innovative nature of the gradual implementation of the model in its structures, adapting to the demands of society as a global framework in which it evolves and to which, to a certain extent, it serves or provides a service from a mostly business point of view.

The documentary scrutiny of the models presented and their results in other countries, lead us to think about their implementation at the University of Oviedo for the benefit of the region's talent, a competent and trained labour force, which may satisfy the demand for current positions in the different areas and may launch new business ideas that, without a doubt, may be encouraged by the possibility of relocation of many jobs, the acquisition of vision for entrepreneurship and the export of products and services, among others (Álvarez-Arregui, 2019). Being aware of the fact that innovation affects all spheres of life, the implementation of new approaches or models in the educational system may be necessary and imminent; if traditional jobs disappear as they are known, and new occupations appear requiring new skills, universities should adapt and change their curricula, maintaining their prestige as institutions of higher knowledge, introducing new knowledge and experiences, even if this should be to the detriment of some traditional subjects.

New knowledge and experience do not only occur in universities (Arreguit \& Hugues, 2019), but also in industry and in public and social organizations; all of them must get involved under a real approach of dual and continuous education that allows to move at the pace of innovation, it is necessary to engage these spheres to face current and future works and challenges. Further research would be to study the skills that the working environment in Asturias wants for future employees and to analyse these results on the basis of an OTA-type student/employee model, in order to obtain sustainable data about the importance of implementing actions in the University supported under this model and, therefore, catalysts for the talent demanded by the markets.

Companies and organizations also have to promote changes in their structures in order to remain in the market. But, to do so, they continuously refer to the need to hire prepared people, and remark the lack of talent, the difficulties in finding employees with soft skills, with leadership, with competences that are not only instrumental but also generic and specific (Jericó, 2008). This is the main reason for the growing increase in training plans in companies, aimed at improving management and teamwork skills.

The capacity to acquire new knowledge will be more valuable than one's own knowledge in the future, perhaps because it is time to unlearn what has been learned in order to learn again. Waiting without intervening is no longer an option; being aware of the fact that the necessary change is above all cultural, with everything that this entails, and sharing a vision with Álvarez-Arregui \& Arreguit (2019) and Arreguit \& Hugues (2019), we propose to explore in depth the possibilities of the OTA model in the university environment, as the Instituto de Investigación e Innovación Educativa (Institute for Educational Research and Innovation, INIE) of the University of Oviedo has been doing, because we consider it able to improve the employment of the future. 


\section{Referencias / References}

Álvarez Morán, S., Pérez Collera, A., Suárez Álvarez, M. L. (2008). Hacia un enfoque de la educación en competencias. Madrid: Consejería de Educación y Ciencia. Dirección General de Políticas Educativas y Ordenación Académica.

Álvarez-Arregui, E. (2017). Evolución de la Universidad en la Sociedad del Aprendizaje y la Enseñanza. El valor de las competencias en el desarrollo profesional y personal. Aula Abierta, 48(4), 349-372.

Álvarez-Arregui, E., Arreguit, X. (2019). El futuro de la Universidad y la Universidad del Futuro. Ecosistemas de formación continua para una sociedad de aprendizaje y enseñanza sostenible y responsable. Aula Abierta, 48(4), 447-480.

Arreguit, X., Hugues, J. F. (2019): Competencias y educación para los trabajos y desafíos del mañana: La perspectiva de una empresa. Aula Abierta, 48(4), 373-392.

Baigorri Ruiz, E. (2018). Desarrollo de la competencia transversal de comunicación y relación interpersonal, a través de la gestión del talento en los alumnos de grado de enfermería. Universidad Complutense de Madrid.

Becker, G. (1983). Un análisis teórico y empírico referido fundamentalmente a la educación. Madrid: Alianza.

Berguer, S. L. (1997). Giftedness and gifted: what's it all about? Documento de la base de datos ERIC (ERIC - EC Digest No. E-476).

Centro de Investigaciones Sociológicas (CIS), (2020). http:// www.cis.es/cis/opencms/ES/2 bancodatos/. Accessed 21 May 2020.

Damon, W. (2009). The path to purpose how young people finds their calling in life. New York: Free Press.

Dawson, R. (2013). Building success in the future or work: T-shaped, Pi-shaped and Combshaped Skills. https://rossdawson.com/blog/building-future-success-t-shaped-pishaped-\&-comb-shaped-skills/. Accessed 24 April 2020.

De la Torre, S. (2010). Innovación y aprendizaje como valor y actitud ante la vida. Educar con otra conciencia. En L. Cacheiro; C. Dominguez y A. Medina (coord.). Investigación e innovación de la docencia universitaria en el Espacio Europeo de Educación Superior, (pp. 320-350). Madrid: Fundación Ramón Areces.

De la Torre, S., Pujol, M. (coord.) (2009). Educar con otra conciencia. Una mirada ecoformadora y creativa de la enseñanza. Madrid: Davinci Continental.

Deloitte, P. (2018). El rediseño de la empresa del futuro: eficiencia y rentabilidad 2025. Madrid: Deloitte.

Dormehl, L. (2018). Thinking machines: The inside story of artificial intelligence and our race to build the future. London: WH Allen.

Friedman, T. L. (2016). Thank you for being late: An optimist's guide to thriving in the age of accelerations. New York: Farrar, Straus and Giroux.
Goleman, D. (1996). La Inteligencia Emocional. Madrid: Ed. Kairós. Griffiths, T., Guile, D. (2003). A connective model of learning: The implications for work process knowledge. European Educational Research Journal, 2, 56-73.

Grossi Sampedro, B. A. (2012). Educación emprendedora y educación superior. Estudio de la percepción de los estudiantes de Ciencias Sociales y Jurídicas de la Universidad de Oviedo. Universidad de Oviedo (Asturias).

Heikkinen, K. P. (2018). Exploring studio-based higher education for T-shaped knowledge workers, case LAB studio model. Universidad de Oulu (Finlandia).

Hernández Lechuga, T. G. (2017). Factores determinantes para la retención del talento internacional en la empresa. El Caso de México y España. Universidad Pontificia de Comillas (Madrid).

Instituto de Estudios Superiores de Empresa (2019). El futuro del empleo y las competencias profesionales del futuro: la perspectiva de las empresas. Madrid: IESE.

Jericó, P. (2008). La Nueva Gestión del Talento: construyendo compromiso. Madrid: Pearson Educación, S.A.

Jericó, P., Botín, A. P., Medina, J. (2008). La nueva gestión del talento humano: Construyendo compromiso. Madrid: Prentice Hall.

Lorenzo García, R. (2006). A qué se le denomina talento. Estado del arte acerca de su conceptualización. Revista Intangible Capital, 11(2), 72-163.

McClelland, D. (1973). Testing for competence rather than "intelligence". American Psycholgyst, 28(1), 1-14.

Mertens, L. (1998). Competencias claves. Madrid: OEI.

Pérez-Escoda, N., Sabariego Puig, M. (2015). Valoración de la efectividad de la formación en competencias emocionales en educación superior. En AIDIPE (Ed.), Investigar con y para la sociedad (Vol.2, pp.965-976). Cádiz: Bubok.

Schultz, T. (1992). Restablecimiento del equilibrio económico: los recursos humanos en una economía en proceso de modernización. Barcelona: Gedisa.

Short, E. (ed.) (1984). Competence: inquiries into its meaning and acquisition in educational settings. New York: University Press of America.

Sisk, D. K. (1993). International Handbook of research and development of giftedness and talent. New York: Pergamon Press.

Tansley, C. (2011). What do we mean by the term \talent in talent management? Industrial and Commercial Training, 43(5), 266-274.

Tourón J. (2017). Alta capacidad y desarrollo del talento: aspectos críticos. Talento Educación Tecnología. [Internet]. Marzo 2015. [Accessed 24 May 2017]. Available at http://www.javiertouron. es/2015/03/alta-capacidad-y-desarrollo-deltalento.html

VICongreso de Factor Humano (Human Factor Congress), organised by IESE-IFAES (May 2019), Madrid. https:/ / elpais.com/ economia/2017/03/27/actualidad/1490624613 488525. html. Accessed 22 May 2020. 